\title{
About One Method of On-Line Signature Verification Using Radial Basis Function
}

\author{
Ellina Sergeevna Anisimova ${ }^{1} \&$ Rinat Rivkatovich Ibatullin ${ }^{1}$ \\ ${ }^{1}$ Kazan Federal University, Elabuga, Russia \\ Correspondence: Ellina Sergeevna Anisimova, Kazan Federal University, 89, Kazanskaya Street, Elabuga \\ 423600, Russia.
}

Received: October 7, 2014

Accepted: October 10, $2014 \quad$ Online Published: December 7, 2014

doi:10.5539/mas.v9n1p137

URL: http://dx.doi.org/10.5539/mas.v9n1p137

\begin{abstract}
The article is devoted to the study of a possibility of on-line signature verification using the wavelet transform with the radial basis function. Representation of the signature in the form of function, invariant concerning a position, - the signature is replaced by a broken curve and is described using the angles between the adjacent links - is offered. Using the wavelet transforms for the signature description, comparison of expansion coefficients of functions of signatures by the radial basis function to verify signatures is described in detail. In the result of application of the offered method the magnitude of error of first kind has made $4.4 \%$, the magnitude of error of second kind $-2.8 \%$.
\end{abstract}

Keywords: on-line signature, verification, wavelet transform, radial basis function

\section{Introduction}

Despite existence of a large number of methods for biometric identification of a personality (Ortega-Garcia, et al., 2002), using the signature for this purpose has widespread application (Jain et al., 2002).

Originally the signature (Plamondon, \& Lorette, 1989) was considered as a graphic object only (the static signature) but when appearing the new input equipment (graphic tablets, smartphones) a problem of on-line signature verification arose (Plamondon, \& Srihari, 2000), that is of signature together with dynamics of its creation (the dynamic signature, the graphic tablet or smartphone is used for input (Sayeed, et al., 2010).

The systems of a personality identification, working with the "live" signatures (Maiorana, 2010) are constructed on the analysis of dynamics of the signature reproduction (Gupta and Joyce, 2007), (Leus, 2009) in one, two or three coordinates $(X(t)$ - the movement of a pen on axis $X, Y(t)$ - the movement of a pen on axis $Y, Z(t)-$ intensity of pen pressing).

Having received the signature samples, the verification system expands dependences $X(t), Y(t), Z(t)$ of each signature to series. For this purpose, as a rule, the discrete Fourier transform is calculated (Anisimova, 2014), Walsh and Haar functions can be also used. The statistical processing of coefficients is made, series of average values, which become reference, is calculated. The standard deviations (or distances) from them, necessary to establish a threshold of discrepancy of the new signature to the original, are defined.

In the authentication mode the signature is expanded to series at first, then the received series is compared to a basic reference standard (Vorontsov, 2011). If the threshold of discrepancy is not exceeded, the signature is considered valid (Liwicki, et al., 2011), authentication is confirmed.

The one-coordinate systems of signature verification, constructed by accounting of any of time functions $X(t)$, $Y(t), Z(t)$, provide probability of errors of first and second kind at the level of 0.1 . The two-coordinate systems allow to reach the level of probability of errors about 0.01 . The most complex systems, using the full three of functions, provide the level of probability of errors of first/second kind 0.003 (Ivanov, 2005).

However, the problem of signature verification cannot be considered as finally solved (Nanni, \& Lumini, 2005). As shown in work Unser and Blu, 2002, when studying the non-homogeneous signals the wavelet transform with nonorthogonal radial basis function appears as a rather powerful tool. It is resistant to displacement and allows to receive the integral characteristic of the signal. Besides, the Fourier transform, used in the problems of verification, does not consider dynamics of the signal, it allows to receive only information on the frequency 
present at the signature signal. In this regard the aim of this work is to study a possibility of on-line signature verification using the wavelet transform with radial basis function.

The novelty of the offered method of signature verification:

- representation of the signature in the form of function, invariant concerning a position;

- $\quad$ expansion of the found function by the radial basis function of a special kind.

\section{Methods}

\subsection{Wavelet Transform with the Radial Basis Function}

\subsubsection{Representation of the Signature in the Form of Function}

Transition from the signature to its characteristic (Ibrahim, et al., 2009) must keep its properties but must not depend on the position of the signature and its orientation. The on-line signature (Wang, \& Zhang, 2013) is presented in the form of a text file each record of which is vector $p_{k}=\left(x_{k}, y_{k}\right)$ plotted by the coordinates of the next point (force of pressing was not considered). As the position of the next point was defined at equal intervals of time in the specified description dynamics (Rioja, et al., 2004) of the signature was considered.

At verification (Huang, \& Yan, 2003), comparison of the current signature with a basic reference standard the following problems arise:

1) dependence of the signature on the position on the tablet;

2) variability of the signature (length-variability and other changes).

To solve the first problem - exclusion of dependence on the position on the screen - to replace the signature by some broken curve and to describe it using the angles between the adjacent links is offered. The magnitude of the angles between the adjacent links does not depend on the position of the signature on the page.

For this purpose the signature file is transformed to the values of function $f(t)$ by the following rule:

- $\quad$ the number sequence is constructed $t_{k}, k=0,1, \ldots$, where $t_{0}=0, t_{k}=\left|p_{k+1}-p_{k}\right|, k>0 ; p_{k}=\left(x_{k}, y_{k}\right)$ - vector plotted by the coordinates of the next point;

- $\quad f_{0}=0, f_{k}=f\left(t_{k}\right)-$ angle between vectors $p_{k}$ and $p_{k+1}$.
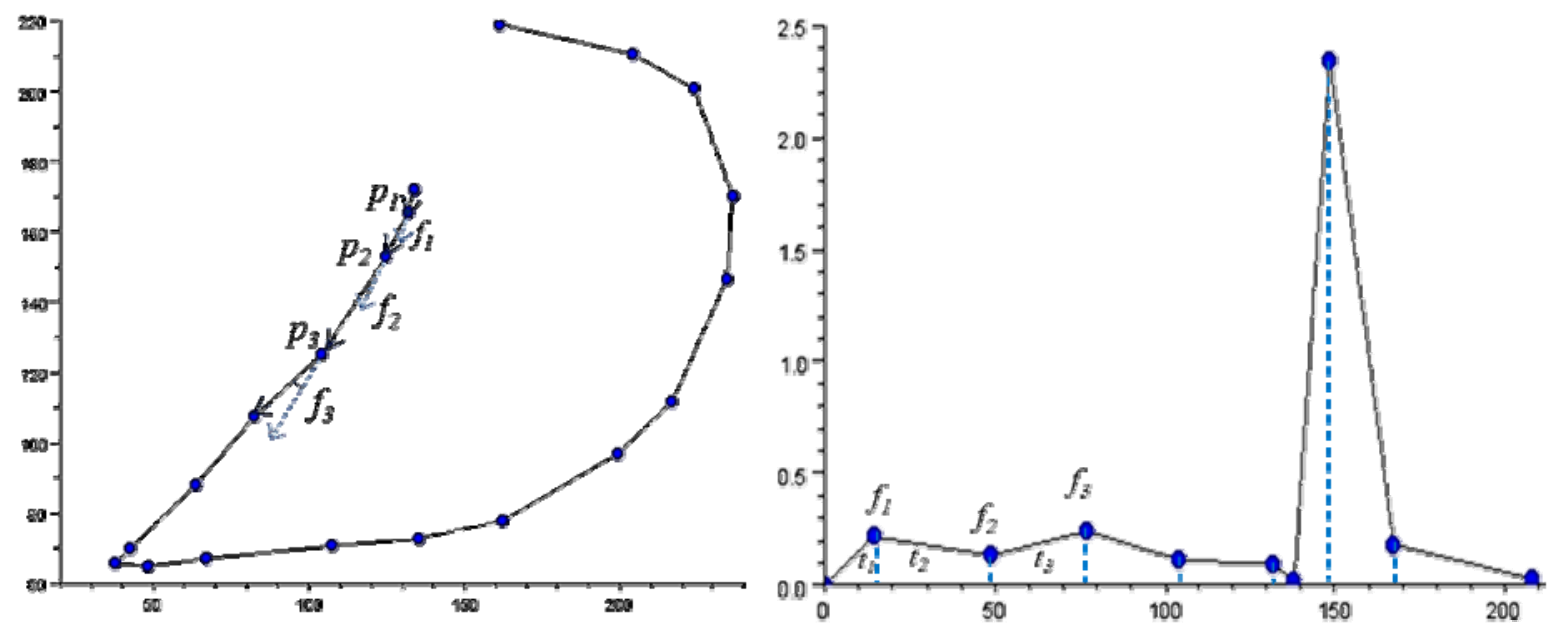

Figure 1. Representation of the signature in the form of function

Signatures of the same person are not absolutely identical. In particular, the constructed functions will have different lengths (Faundez-Zanuy, 2005). Therefore, to have an opportunity to compare such functions later on, the signature functions are led to standard length.

For this purpose the domain of each function is broken into the same number $(\mathrm{N})$ of points $x_{1}, x_{2}, \ldots, x_{N}$, equidistant from each other and values of functions in these points are calculated by a spline interpolation method (Unser, 1999).

2.1.2 Using Radial Functions and Wavelets for Signature Verification

The problem was reduced to comparison of functions which are defined on the same set of arguments. To 
characterize a function in general, not in separate points, the expansion of functions by the basis is used. Comparison of functions is reduced to comparison of the obtained expansion coefficients.

In this work the approach, presented in Unser and Blu, 2002, was used. This approach is based on using nonorthogonal radial basis function to expand the signature function. Due to a choice of the radial basis function the behaviour of the signature function in the point is expressed through the values in other points by what the integral characteristic of the signature is reached.

Approximation by the radial basic function has a form:

$$
\sum_{k=1}^{N} a_{k} \rho\left(\left\|x_{j}-x_{k}\right\|\right)=f_{j}, \quad 1 \leq j \leq N
$$

where:

$$
\begin{gathered}
\left\|x_{j}-x_{k}\right\|-\text { Euclidean distance between } x_{j} \text { and } x_{k} \text { nodes; } \\
\rho-\text { radial function; } \\
a_{k}-\text { weighting coefficient; } \\
f_{j}=f\left(x_{j}\right)-\text { value of function (magnitude of the angle) in the point } x_{j} .
\end{gathered}
$$

Radial function:

$$
\rho(x)=x^{\alpha}
$$

where: $0<\alpha<1$

Here function $f$ is presented in the form of the sum $\mathrm{N}$ of the radial basic functions with centres $\left\{x_{k}\right\}_{k=1}^{N}$ and weighting coefficients $\left\{a_{k}\right\}_{k=1}^{N}$.

We see that (1) represents convolution of the wavelet function $\rho$ (as the wavelet function the radial function is taken) with signal $a_{k}$, so this is a wavelet transform. It transfers the assumed function to a form which makes some of its values more amenable to study, allows to receive a high compression ratio in combination with a good quality of the reconstructed signal (Unser, \& Blu, 2000), (Astafyeva, 1996).

Signs of the signature are ratios $\left\{a_{k}\right\}_{k=1}^{N}$ of representation of the signature function by means of the radial basis function.

For each of copies of signatures we shall construct and solve the system of linear equations:

$$
\left\{\begin{array}{c}
a_{1} \rho(0)+a_{2} \rho\left(\left\|x_{1}-x_{2}\right\|\right)+\cdots+a_{N} \rho\left(\left\|x_{1}-x_{N}\right\|\right)=f\left(x_{1}\right) \\
a_{1} \rho\left(\left\|x_{2}-x_{1}\right\|\right)+a_{2} \rho(0)+\cdots+a_{N} \rho\left(\left\|x_{2}-x_{N}\right\|\right)=f\left(x_{2}\right) \\
\cdots \\
a_{1} \rho\left(\left\|x_{N}-x_{1}\right\|\right)+a_{2} \rho\left(\left\|x_{N}-x_{2}\right\|\right)+\cdots+a_{N} \rho(0)=f\left(x_{N}\right) .
\end{array}\right.
$$

As $\rho(x)$ according to Unser and Blu, 2002 we shall take the symmetric (radial) central basic function

$$
\rho(x)=|x|^{\alpha}
$$

where:

$$
0<\alpha<1
$$

From each system of equations we shall define coefficients $\left\{a_{k}\right\}_{k=1}^{N}$ of expansion by means of the radial basis function and construct their diagrams (Figure 2): 


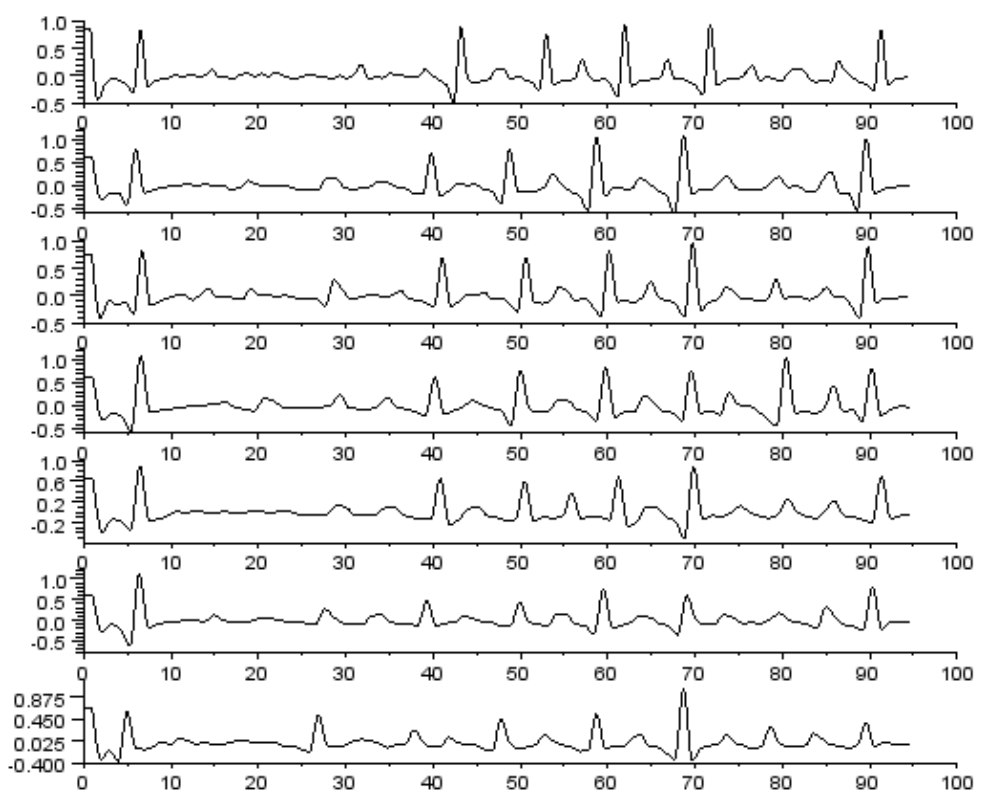

Figure 2. Diagrams of coefficients $\left\{a_{k}\right\}$ at $0<\alpha<1(\alpha=0.3)$

We see that on diagrams $\left\{a_{k}\right\}_{k=1}^{N}$ of copies of one writer's signature at $0<\alpha<1$ a certain regularity and stability of values in some points is observed.

For comparison let us consider $\rho(x)=|x|^{\alpha}$, where $\alpha=1$, i.e. $\rho(x)=|x|$ (Figure 3):

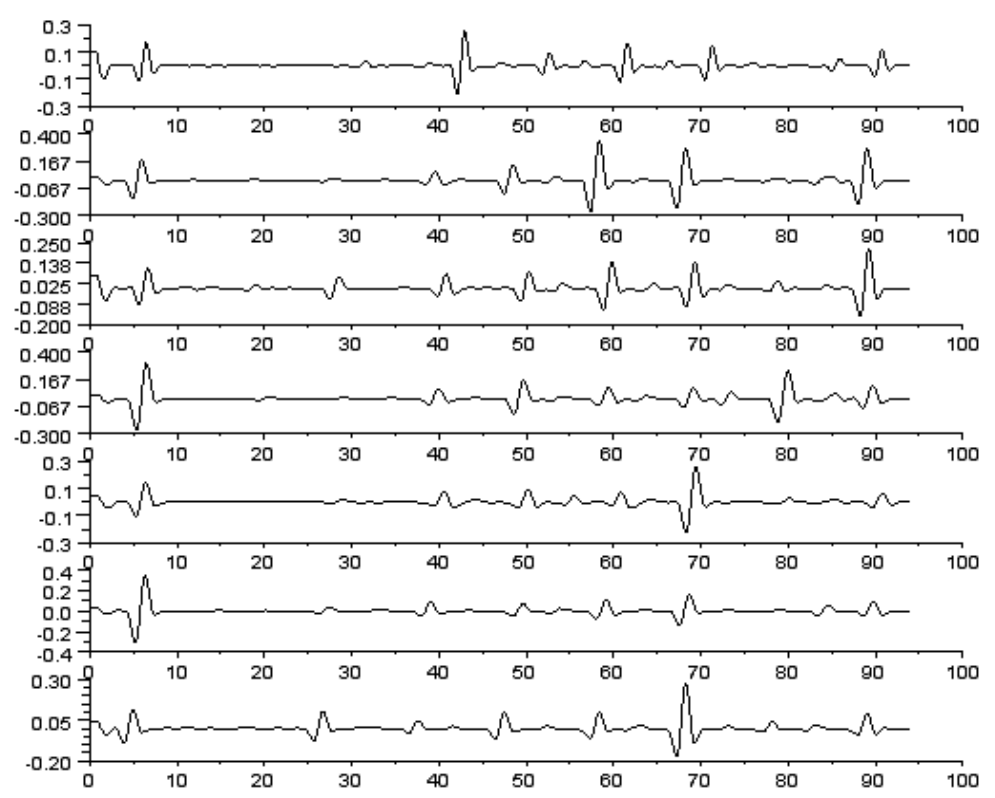

Figure 3. Diagrams of coefficients $\left\{a_{k}\right\}$ at $\alpha=1$

In this case regularity is also traced but already not so clearly as at $0<\alpha<1$.

A question arises how many points it is necessary to use for each copy? We shall find it out experimentally. Let $\mathrm{N}_{\text {min }}$ be a minimum quantity of points of the signature among the copies of the writer's signature, $\alpha=0.001$. At $\mathrm{N}<\mathrm{N}_{\text {min }}$ (in this case the minimum quantity of points of the signature is equal 95) we shall receive (Figure 4-5): 


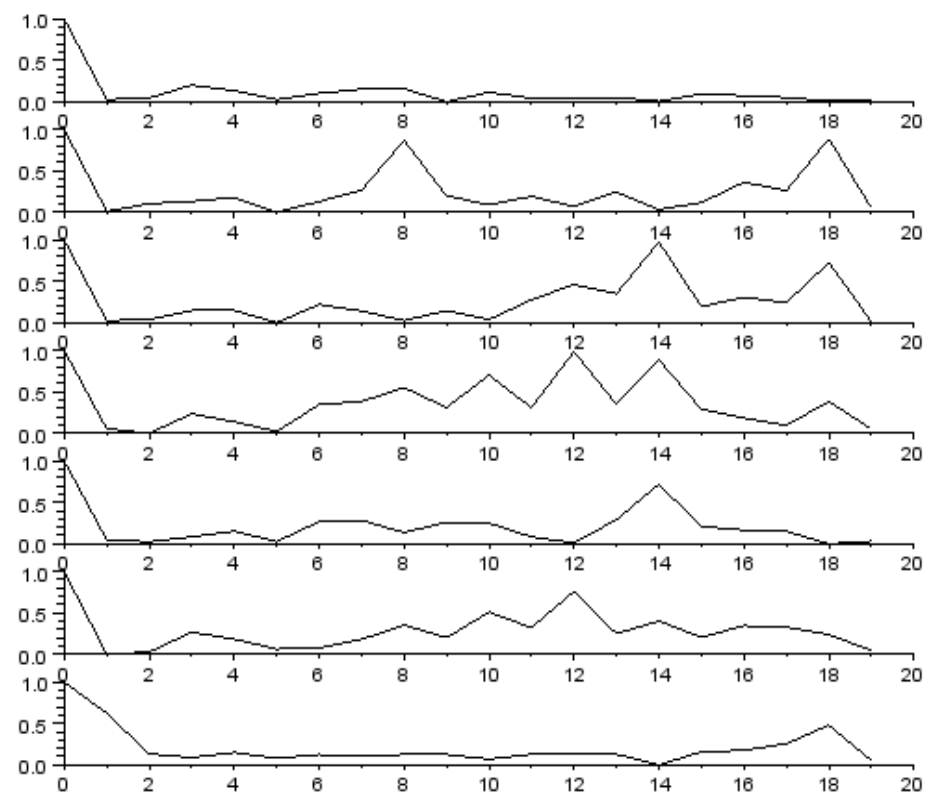

Figure 4. Diagrams of coefficients $\left\{a_{k}\right\}$ at $\mathrm{n}=20(k=\overline{1, n})$

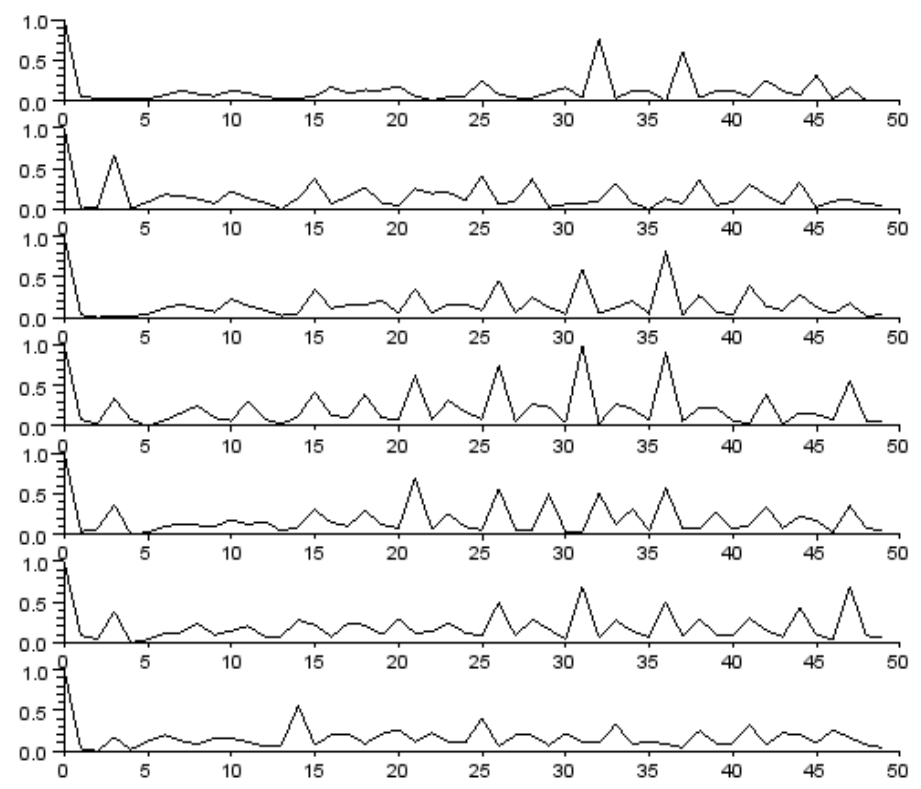

Figure 5. Diagrams of coefficients $\left\{a_{k}\right\}$ at $\mathrm{n}=50(k=\overline{1, n})$

If to compare to Figure 2, where $\mathrm{N}=\mathrm{N}_{\min }$ - the minimum quantity of points on a set of copies of the signature, we can note that at $\mathrm{N} \geq \mathrm{N}_{\min }$ the more exact picture is observed. Really, at reduction of quantity of points the information on the signature is lost. The quantity of points on each copy of the signature is unequal. Therefore, to save the information as $\mathrm{n}$ at least the minimum number of points of the signature among all the copies of the signature should be taken.

We shall enter metrics for coefficients $\left\{a_{k}\right\}_{k=1}^{N}$. For this purpose we shall define the average values $\left\{\overline{a_{k}}\right\}_{k=1}^{N}$ for all the copies of the writer's signature at first. Then we shall calculate distance from the "averaged" vector $\left\{\overline{a_{k}}\right\}_{k=1}^{N}$ of the writer's signatures to the vector of coefficients $\left\{a_{k}\right\}_{k=1}^{N}$ of each copy of his signatures.

During the studies it was found out that the distance between the vectors of the signatures, made by different people, exceeds the distance between the vectors of one writer's signatures. This fact opens an opportunity for 
verification of signatures by application of the wavelet transforms and radial basis function and by further comparison of the signatures taking into account the entered metrics.

It is possible to enter a certain threshold when comparing the distances between the vectors of the signatures. If the distance between the vectors of signatures $\mathrm{A}$ and $\mathrm{B}$ is below the threshold a decision on identity of signatures $\mathrm{A}$ and $\mathrm{B}$ is made, if the distance between the vectors of signatures $\mathrm{A}$ and $\mathrm{B}$ is above the threshold it is considered that they belong to different writers.

\subsection{Time-Frequency Windowed Fourier Transform of the Signature Signal}

For comparison we shall consider also the traditional way of verification of signatures using the Fourier transform.

So, the on-line signature is presented in the form of a text file each record of which is vector $p_{n}=\left(x_{n}, y_{n}\right), n=0 . . N-1$, plotted by the coordinates of the next point of the signature.

Let us consider the on-line signature in the complex plane. For this purpose we shall construct the sequence of complex numbers $\left\{z_{n}\right\}, n=0 . . N-1$, of kind:

where $\left(x_{n}, y_{n}\right)$ - coordinates of the next point.

$$
z_{n}=x_{n}+i \cdot y_{n}
$$

To the received sequence $\left\{z_{n}\right\}$ the complex Fourier transform can be applied:

$$
F_{k}=\sum_{n=0}^{N-1} z_{n} e^{\frac{-2 \pi i k n}{N}}, k=0 . . N-1,
$$

where $N$ - quantity of points of the entered signature, $k$ - number of the current counting of the Fourier transform, $F_{k}$ - complex amplitudes of the sinusoidal signals composing the original signal.

The received function can be used for the signature study. But the Fourier transform has a significant disadvantage - it gives information only on the frequency, which is in the signal, and does not give any information in what time interval this frequency is in the signal. As we study the dynamic signature, it is more correct to use another tool of the spectral analysis for its study - the windowed Fourier transform. The time-frequency windowed Fourier transform is a variety of the Fourier transform and is defined as follows:

$$
F(w, m)=\sum_{n=-\infty}^{\infty} z_{n} w_{n-m} e^{-i w n}
$$

Here $w_{n-m}-$ the window function of the transform displacement, $m$ - the fixed values of the displacement. As the Transform window both a rectangular window and special weight windows (Bartlett window, Hamming window, Gaussian window, etc.) can be used.

A result of the Windowed Fourier Transform is not spectrum of the original signal but spectrum of signal performing and the window function. Spectrum, received by means of the Windowed Fourier Transform, is an assessment of spectrum of the original signal. The time-frequency windowed Fourier transform allows to receive the characteristic of distribution of the signal frequency (with amplitude) in time.

Let us conduct the study of the on-line signature in the complex plane applying the time-frequency windowed Fourier transform. As the window function we shall take Hamming window with the window width $\mathrm{N}=16$.

The spectrogram of the signature signal, received by means of the Windowed Fourier Transform, will have the following form: 


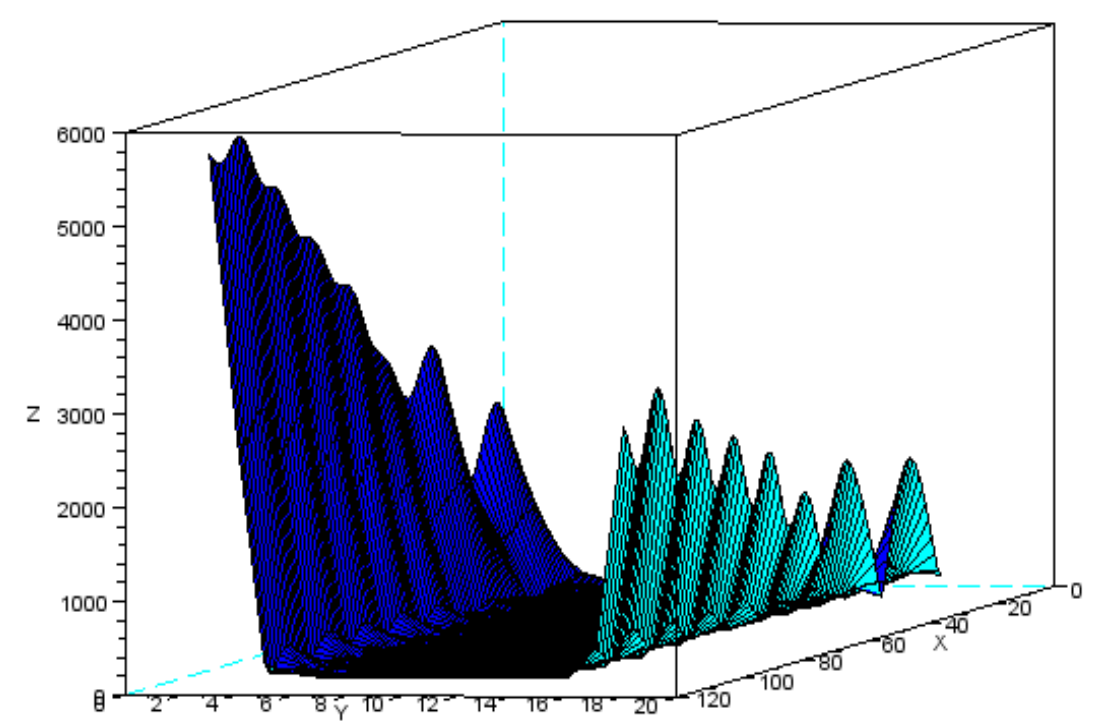

Figure 6. Spectrogram of the signature signal

Here $\mathrm{X}$ - time (displacement), $\mathrm{y}$ - frequency (the coefficient number), $\mathrm{Z}$ - amplitude (the coefficient module).

Formally, the received signature function (function of two variables) can be used for the signature description but individuality is appeared depending on the values of this function in different points. For this purpose to search for such dependence the approach, presented in Unser, Blu, 2002, was used.

Let us apply the wavelet transform with the radial basis function to the signature function:

$$
\sum_{i=1}^{N} \sum_{j=1}^{M} a_{i j} \rho\left(\left\|z-z_{i j}\right\|\right)=F
$$

where: $\left\|z-z_{i j}\right\|$ - Euclidean distance between points $z=(x, y)$ and $z_{i j}=\left(x_{i}, y_{j}\right) ; i=1 \ldots N-$ time;

$j=1 \ldots M-$ frequency;

$\rho\left(\left\|z-z_{i j}\right\|\right)=\left\|z-z_{i j}\right\|^{\alpha}$ - radial function, $0<\alpha<1$;

$\left\{a_{i j}\right\}_{i=1 . . N ; j=1 . . M}-$ matrix of the weighting coefficients;

$F=F(z)-$ function value in point $z=(x, y)$.

Here function $F$ is presented in the form of the sum NxM of the radial basis functions with centres $\left\{z_{i j}\right\}_{i=1 . . N ; j=1 . . M}$ and weighting coefficients $\left\{a_{i j}\right\}=\left\{a_{i j}\right\}_{i=1 . . N ; j=1 . . M}$.

Signs of the signature are coefficients $\left\{a_{i j}\right\}$ of representation of the signature function by means of the radial basis function. For each of copies of signatures we shall construct and solve the system of linear equations (7) concerning coefficients $\left\{a_{i j}\right\}$. 


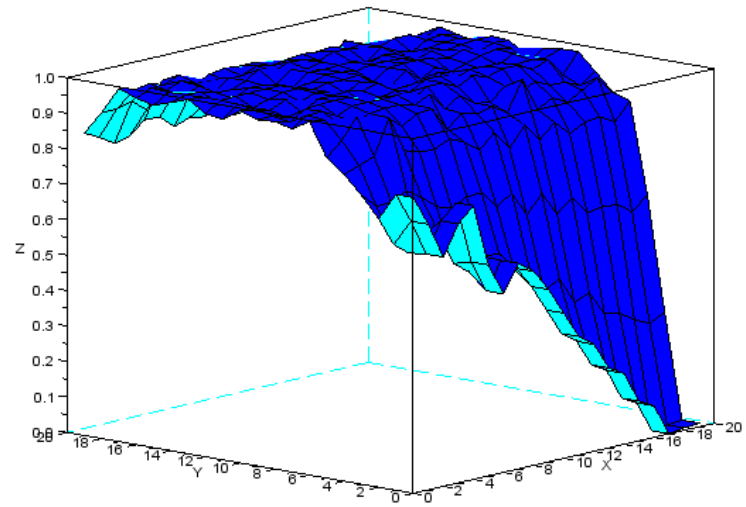

(a)

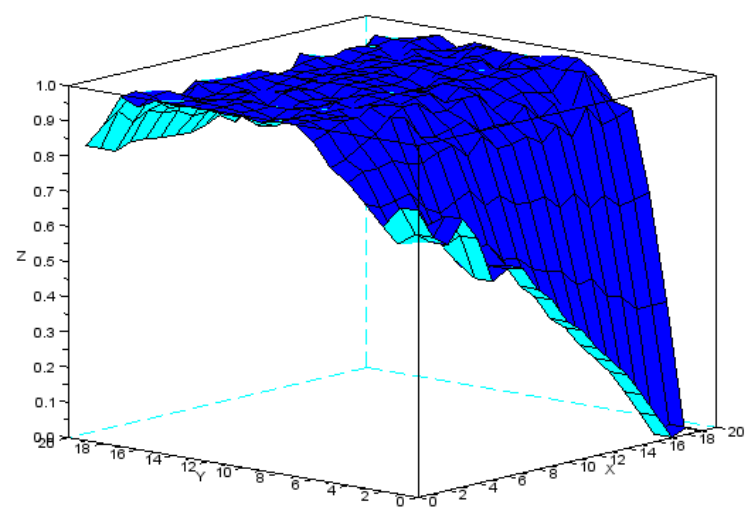

(c)

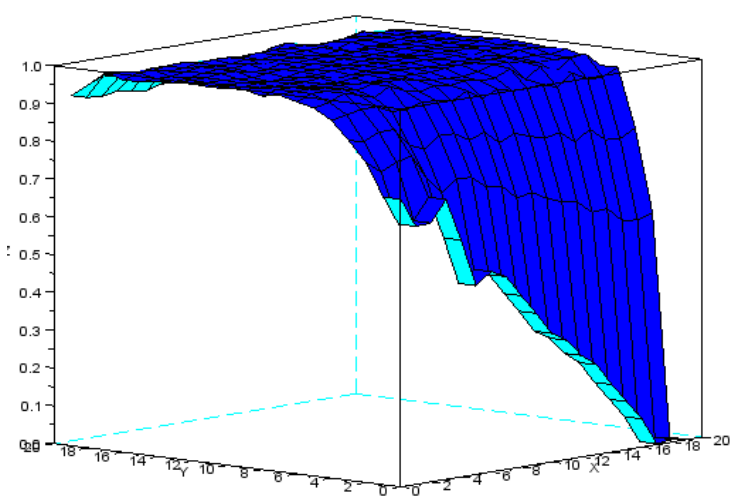

(b)

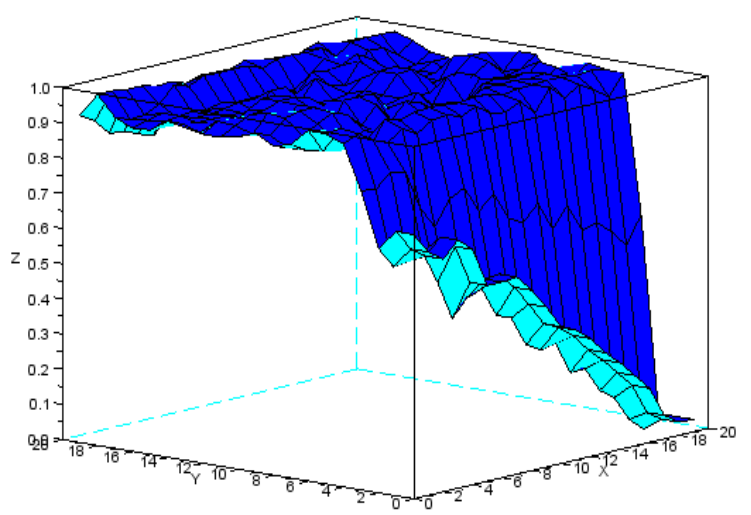

(d)

Figure 7. Surface (a) - (d), the obtained values of the matrix coefficients $\{$ aij $\}(\alpha=0.001)$ multiple instances of the signature of one of the participants in the experiment

We construct a surface corresponding to the coefficients \{aij\} multiple instances of the signature of one of the participants in the experiment. When constructing the surfaces we shall make normalization of the values before to have a possibility of their further comparison. See (Fig. 7) that the image surfaces corresponding instances of the signature of the same author, are similar; they exhibit a certain regularity and stability of value at some point. We shall construct the surfaces for other experiment participants and then carry out an inspection of verification reliability.

Let us enter metrics for coefficients $\left\{a_{i j}\right\}$. For this purpose we shall define matrix of average values $\left\{\overline{a_{i j}}\right\}$ for all the copies of the writer's signature. Next we shall calculate the distance from the "averaged" matrix $\left\{a_{i j}\right\}$ of the writer's signatures to matrix of coefficients $\left\{a_{i j}\right\}$ of each copy of his signature.

During the studies it was also found out that the distance between matrixes of coefficients of the signatures, made by different people, exceeds the distance between matrixes of coefficients of one writer's signatures. It is possible to enter a certain threshold when comparing the distances between matrixes of coefficients. If the distance between matrixes of coefficients of signatures $A$ and $B$ is below the threshold, the decision on identity of signatures $A$ and $B$ is made, if the distance between matrixes of signatures $A$ and $B$ is above the threshold, it is considered that they belong to different writers.

\section{Results}

The experiment on the on-line signature verification has been conducted on 50 participants. For each participant 6-7 copies of signatures have been used.

Let us consider the process of identification of one of the experiment participants. We shall divide all the available signatures (the writer's signatures and other participants' ones) into 2 classes before:

1 class - writer's signatures; 


\section{2 class - other participants' signatures.}

We shall make comparison of two constructed classes (Gruber, et al., 2010) i.e. shall compare the writer's signatures and other participants' signatures.

At identification by means of the wavelet transform with the radial basis function vector of coefficients $\left\{a_{k}\right\}=\left\{a_{k}\right\}_{k=1}^{N}$ of expansion by means of the radial basis function corresponds to each signature. We shall calculate the distances between the averaged vector of coefficients $\left\{\overline{a_{k}}\right\}$ of the writer's signatures (averaged vector of 1 class) and vectors of coefficients $\left\{a_{k}\right\}$ of other signatures (his ones and other participants' signatures - vectors of 1 and 2 classes). We shall form vector of distances $\left(r_{l}, r_{2}, \ldots, r_{s}, r_{s+1}, \ldots, r_{n}\right)$, where $r_{l}, r_{2}, \ldots, r_{s}-$ distances between vectors of coefficients $\left\{a_{k}\right\}$ of 1 class (1..s - numbers of signatures of 1 class) and averaged vector of coefficients $\left\{a_{k}\right\}$ of 2 class and averaged vector of coefficients $\left\{\overline{a_{k}}\right\}$ of the writer.

To represent the results of identification we shall construct a ROC curve (Receiver Operator Characteristic) (Paklin, 2013). The ROC curve is often used to represent the results of binary classification in machine learning. As there are two classes, one of them is called as a class with positive outcomes (we shall designate the writer's signatures, 1 class), the second one - with negative outcomes (other participants' signatures, 2 class).

The ROC curve (Paklin, Oreshkov, 2013) shows dependence of the quantity of correctly classified positive examples on the quantity of incorrectly classified negative examples. In ROC analysis terminology the first examples are called truly positive set, the second ones - false negative set. At that it is supposed a qualifier has some parameter (threshold), varying which, we shall receive one or another dividing into two classes.

Depending on it the different magnitudes of errors of I and II kind shall be made (Gorelik, Skripkin, 1977). The error of first kind is probability to take the writer's signature for the false one. The error of second kind is equal to probability to take the false signature for the writer's signature.

So, $\left\{r_{s}\right\}_{s=1}^{n}$ - vector of the distances, where $n$ - total number of all the participants' signatures. Let us define minimum $r_{\text {min }}$ and maximum $r_{\max }$ values of the elements of vector $\left\{r_{s}\right\}_{s=1}^{n}$. We shall break the jog $\left[r_{\min } ; r_{\max }\right]$ up into $M$ equal parts with step $h=\frac{r_{\max }-r_{\min }}{M}-$ we shall receive vector $\left\{\tau_{i}\right\}_{i=0}^{M}$, where $\tau_{0}=r_{\min }, \tau_{M}=r_{\max }, \tau_{i+1}=\tau_{i}+h$.

For each value of threshold $\tau_{i}, 0 \leq i \leq M$ :

1) we define the signatures for which the corresponding value of distance $r_{s}$ less than $\tau_{i}$ and check whether they are really the writer's signatures. We define the value of sensitivity - ratio of numbers of the correctly classified writer's signatures to the total number of the writer's signatures;

2) we define the signatures for which the corresponding value of distance $r_{s}$ more than $\tau_{i}$ and check whether they are really the signatures not belonging to the writer. We define the value of specificity - ratio of numbers of the correctly classified signatures, not belonging to the writer, to the total number of other participants' signatures.

So, we form the ROC curve: we define a sensitivity vector $\left\{s e_{i}\right\}_{i=0}^{M}$ and specificity vector $\left\{s p_{i}\right\}_{i=0}^{M}$, then on axis $Y$ we put values $\left\{s e_{i}\right\}_{i=0}^{M}$, on axis $X:\left\{1-s p_{i}\right\}_{i=0}^{M}, \quad 0 \leq i \leq M$.

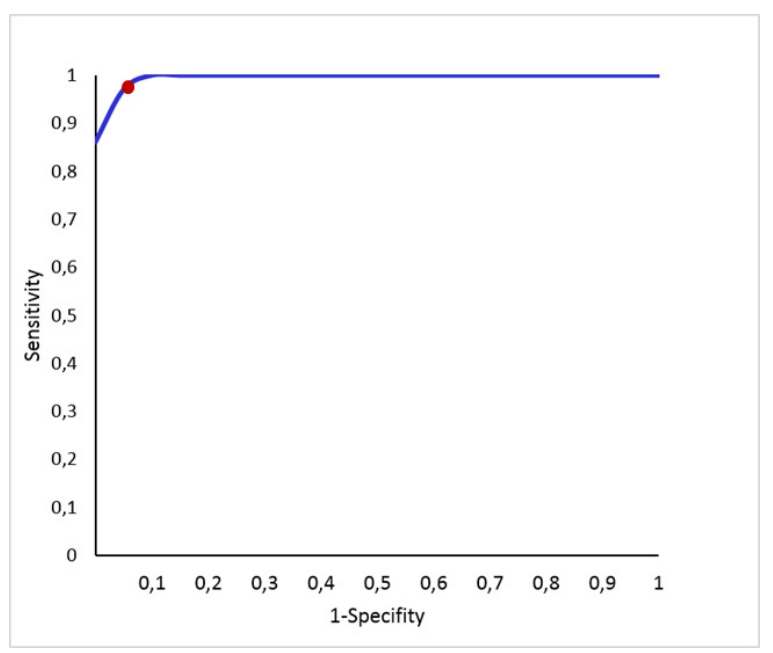

Figure 8. ROC curve of one of the experiment participants 
We shall find the value of threshold for decision-making proceeding from the balance between sensitivity and specificity. According to the chosen value of threshold magnitudes of errors of first and second kind are defined for the signature verification. The value of threshold is defined for each writer individually.

In the result of the conducted experiment the average magnitude of error of first kind has made $4.4 \%$, the magnitude of error of second kind has made $2.8 \%$.

We shall similarly make an experiment on the same collection of signatures by means of the traditionally used time-frequency windowed Fourier transform.

In this case matrix of coefficients $\left\{a_{i j}\right\}$ of expansion by means of the radial basis function corresponds to each signature. We shall calculate the distances between the averaged matrix of coefficients $\left\{a_{i j}\right\}$ of the writer's signatures (of 1 class) and matrixes of coefficients $\left\{a_{i j}\right\}$ of all the signatures (matrixes of 1 and 2 classes). Thus, we shall form vector of distances $\left(r_{1}, r_{2}, \ldots, r_{s}, r_{s+1}, \ldots, r_{n}\right)$, where $n$ - number of signatures, $r_{1}, r_{2}, \ldots, r_{s}-$ distances between matrixes of coefficients $\left\{a_{i j}\right\}$ of 1 class and the averaged matrix of coefficients $\left\{a_{i j}\right\}$ of 1 class and $r_{s+1}, \ldots, r_{n}$ - distances between matrixes of coefficients $\left\{a_{i j}\right\}$ of 2 class and averaged matrix $\left\{a_{i j}\right\}$ of 1 class.

The vector of distances $\left\{r_{s}\right\}_{s=1}^{n}$ has been received, where $n$-total number of all the participants' signatures. On its basis vector $\left\{\tau_{i}\right\}_{i=0}^{M}$ and also sensitivity vector $\left\{s e_{i}\right\}_{i=0}^{M}$ and specificity vector $\left\{s p_{i}\right\}_{i=0}^{M}$ have been formed. We shall find the value of threshold for decision-making also proceeding from the balance between sensitivity and specificity. According to the chosen value of threshold for the signature verification the magnitudes of errors of first and second kind have been defined. The value of threshold is defined for each writer individually.

In the result of the conducted experiment the average magnitude of error of first kind has made $4.4 \%$, the magnitude of error of second kind $-3.6 \%$.

\section{Discussion}

The classical verification (identification) of a person by handwriting (Plamondon et al., 2012) implies comparison of the analyzed image with the original. Such a procedure exactly is made, for example, by a bank operator when compiling documents. Obviously, the accuracy of such a procedure, from the point of view of probability of making a wrong decision, is rather low. Besides, also a subjective factor influences on spread of values of probability of making a correct decision at identification.

Fundamentally new possibilities of verification by handwriting open when using automatic methods of the analysis of handwriting and decision-making. These methods allow to exclude a subjective factor and to reduce probability of errors at decision-making considerably.

In this work a method of on-line signature verification by means of the wavelet transform with the radial basis function has been realized. The signature is represented in the form of function of one argument by means of angles between the adjacent links corners invariant concerning a position on the page. As biometric parameters coefficients $\left\{a_{k}\right\}_{k=1}^{N}$ of representation of the signature function by means of the radial basis function are used.

The distance, calculated between the functions of different writers' signatures, exceeds the distance between the functions of one writer's signatures what opens opportunities for verification of signatures. In the result of the experiment the average magnitude of error of first kind has made $4.4 \%$, the magnitude of error of second kind $2.8 \%$. To represent the results of verification the ROC curves have been constructed for each experiment participant.

Also for comparison the on-line signature verification by means of the Fourier transform with the radial basis function has been carried out. Thanks to using the Windowed Fourier Transform the dynamic characteristics of the signature are taken into account. In the result of application of the time-frequency transform the average magnitude of error of first kind has made $4.4 \%$, the magnitude of error of second kind 3.6\%. As biometric parameters coefficients $\left\{a_{i j}\right\}$ of representation of the signature function by means of the radial basis function are used.

\section{Conclusion}

The analysis of the signature as biometric characteristic taking into account dynamics has been carried out. The method of verification of signatures with application of the radial basis function has been studied. It was found out that the radial functions (3), used as the wavelet-functions, allow to weaken noises and to increase the existing regularities, it means to increase differences of the writer's signatures from copies of other writer' signatures. When using this method dynamics of the signature was taken into account, what defines advantage of this method compared to the classical methods of verification. 
The experiment on verification of on-line signatures using the radial basis function has been conducted on 50 participants. The used collection of signatures corresponds to a rather small organization most of all when carrying out a procedure of verification of signatures. The large companies, carrying out verification of the employees' signatures, as a rule, have collections of signatures of larger volume (Jain, et al., 2002). Therefore, a prospect of this work is realization of this method of verification on a collection of signatures of larger volume. However, even on a collection of signatures of small volume the method has showed extremely good results.

The offered way does not deny the existing verification methods (Kamel, et al., 2008), (Impedovo, \& Pirlo, 2008), (Fabregas, Faundez-Zanuy, 2009), (Miguel-Hurtado, et al., 2007), it is an additional alternative when determining the results of the signature recognition.

\section{References}

Anisimova, E. (2014). On-line signature verification using the Windowed Fourier Transform and Radial Basis Function. Computer Researches and Modeling, 6(3), 357-364.

Anisimova, E. (2014). Signature verification using Radial Basis Function. Basic Researches, 9(6), 1185-1189.

Astafyeva, N. (1996). Wavelet Analysis: bases of the theory and examples of application. Achievements of Physical Sciences, 166(11), 1145-1170.

Fabregas, J., \& Faundez, Z. M. (2009). On-line signature verification system with failure to enroll management. Pattern Recognition, 42, 2117-2126.

Faundez-Zanuy, M. (2005). Biometric recognition: why not massively adopted yet? IEEE Aerosp. Electron. Syst. Mag., 20(8), 25-28.

Faundez-Zanuy, M. (2005). Data fusion in biometrics. IEEE Aerosp. Electron. Syst. Mag., 20(1), 34-38.

Gorelik, A., \& Skripkin, V. (1977). Recognition Methods. Moscow: "Vyssh. school".

Gruber, C., Gruber, T., Krinninger, S., \& Sick, B. (2010). Online signature verification with support vector machines based on LCSS kernel functions. IEEE Transactions on Systems, Man, and Cybernetics, Part B: Cybernetics, 40(4), 1088-1100.

Gupta, G., \& Joyce, R. (2007). Using position extrema points to capture shape in on-line handwritten signature verification. Pattern Recognition, 40, 2811-2817.

Huang, K., \& Yan, H. (2003). Stability and style-variation modeling for on-line signature verification. Pattern Recognition, 36, 2253-2270.

Ibrahim, M., Kyan, M., \& Ling, G. (2009). On-line signature verification using global features. Proceedings of the Can. Conference on Electric. Comput. Engineering. http://dx.doi.org/10.1109/CCECE.2009.5090216.

Impedovo, D., \& Pirlo, G. (2008). Automatic signature verification - state of the art. IEEE Trans. Syst. Man Cybern. Part C Appl. Rev., 38(5), 609-635. http://dx.doi.org/10.1109/TSMCC.2008.923866.

Ivanov, G. (2005). Review of methods of biometric identification of enterprise information network users. Scientific and Technical Bulletin of St. Petersburg State University of ITMO. Modern Technological Solutions, 20, 41-48.

Jain, A., Friederike, D., \& Connel, S. (2002). On-line signature verification. Pattern Recognition, 35(12), 2963-2972.

Kamel, N., Sayeed, \& Ellis, G. (2008). Glove-based approach to on-line signature verification. IEEE Trans. Pattern Anal. Machine Intell, 30, 1109-1113.

Leus, A. (2009). Biometric authentication by signature dynamic characteristics. "Secuteck.ru" - About Security Systems: Catalog "SKUD. Anti-terrorism". Retrieved September 29, 2014, from http://www.secuteck.ru/articles2/sys_ogr_dost/biometrich-autentifikac-po-dinamich-harakter-podpisi

Liwicki, M., Malik, M., Heuvel, C., Xiaohong, C., Berger, C., Stoel, R., Blumenstein, M., \& Found, B. (2011). Signature verification competition for online and offline skilled forgeries (SigComp2011). 2011 International Conference on Document Analysis and Recognition (ICDAR).

Maiorana, E. (2010). Biometric cryptosystem using function based on on-line signature recognition. Expert Systems with Applications, 37, 3454-3461.

Miguel-Hurtado, O., Mengibar-Pozo, L., Lorenz, M., \& Liu-Jimenez, J. (2007). Online signature verification by dynamic time warping and Gaussian mixture models. IEEE $41^{\text {st }}$ Annual Carnahan Conference on Security 
Technology.

Nanni, L., \& Lumini, A. (2005). Ensemble of Parzen window classifiers for on-line signature verification. Neurocomputing, 68, 217-224.

Ortega-Garcia, J., Gonzalez-Rodriguez, J., Simon-Zorita, D., \& Cruz-Llanas, S. (2002). From biometrics technology to applications regarding face, voice, signature and fingerprint recognition systems. Biometrics Solutions for Authentication in an E-World, 12, 289-337.

Paklin, N. (2013). Logistic regression and ROC analysis - mathematical apparatus of BaseGroup Labs. Technologies of the Data Analysis. Retrieved September 29, 2014, from http://www.basegroup.ru/library/analysis/regression/logistic/

Paklin, N., \& Oreshkov, V. (2013). Business Analytics: From Data to Knowledge. SPb.: Peter.

Plamondon, R., \& Lorette, G. (1989). Automatic signature verification and writer identification - the state of the art. Pattern Recognition, 22(2), 107-131.

Plamondon, R., \& Srihari, S. (2000). On-line and Off-line Handwriting Recognition: A Comprehensive Survey. IEEE Trans. PAMI, 22(1), 63-84.

Plamondon, R., O’Reilly, C., Galbally, J., Almaksour, A., \& Anquetil, E. (2012). Recent developments in the study of rapid human movements with the kinematic theory: Applications to handwriting and signature synthesis. Pattern Recognition Letters, 1-11.

Rioja, F., Mryatake, M., Meana, H., \& Toscano, K. (2004). Dynamic features extraction for on-line signature verification. $14^{\text {th }}$ International Conference on Electronics, Communications and Computers.

Sayeed, S., Samraj, A., Besar, R., \& Hossen, J. (2010). Online Hand Signature Verification: A Review. Journal of Applied Sciences, 10(15), 1632-1643.

Unser, M. (1999). Splines: A perfect fit for signal and image processing. IEEE Signal Processing Mag, 16, $22-38$.

Unser, M., \& Blu, T. (2000). Fractional splines and wavelets. SIAM Rev., 42(1), 43-67.

Unser, M., \& Blu, T. (2002). Wavelets, Fractals, and Radial Basis Functions. IEEE Transactions on signal processing, 50(3), 543-553.

Vorontsov, K. (2011). Machine learning (course of lectures). The Information-Analytical Resource Devoted to Machine Learning, Images Recognition and Data Intellectual Analysis. Retrieved September 29, 2014, from http://www.machinelearning.ru/wiki/index.php?title=\%D0\%98\%D0\%BD $\%$ D $1 \% 82 \% \mathrm{D} 0 \% \mathrm{~B} 5 \% \mathrm{D} 1 \% 80 \% \mathrm{D}$ 0\%BF\%D0\%BE\%D0\%BB\%D1\%8F\%D1\%86\%D0\%B8\%D1\%8F_\%D0\%BA\%D1\%83\%D0\%B1\%D0\%B $8 \% \mathrm{D} 1 \% 87 \% \mathrm{D} 0 \% \mathrm{~B} 5 \% \mathrm{D} 1 \% 81 \% \mathrm{D} 0 \% \mathrm{BA} \% \mathrm{D} 0 \% \mathrm{~B} 8 \% \mathrm{D} 0 \% \mathrm{BC} \% \mathrm{D} 0 \% \mathrm{~B} 8 \% \mathrm{D} 1 \% 81 \% \mathrm{D} 0 \% \mathrm{BF} \% \mathrm{D} 0 \% \mathrm{BB} \% \mathrm{D}$ $0 \% \mathrm{~B} 0 \% \mathrm{D} 0 \% \mathrm{~B} 9 \% \mathrm{D} 0 \% \mathrm{BD} \% \mathrm{D} 0 \% \mathrm{~B} 0 \% \mathrm{D} 0 \% \mathrm{BC} \% \mathrm{D} 0 \% \mathrm{~B} 8$

Wang, Y., \& Zhang, Z. (2013). On-line signature verification based on spatio-temporal correlation. Multimed Tools Appl. http://dx.doi.org/10.1007/s11042-013-1408-x

\section{Copyrights}

Copyright for this article is retained by the author(s), with first publication rights granted to the journal.

This is an open-access article distributed under the terms and conditions of the Creative Commons Attribution license (http://creativecommons.org/licenses/by/3.0/). 